\title{
A Case of Capillary Hemangioma of the Nasal Septum in a Patient With Chronic Ischemic Heart Disease and Antiplatelet Treatment Non-Suspendable
}

\author{
Marco Capellia, c, Patrizia Gatti ${ }^{b}$
}

\begin{abstract}
The capillary hemangioma is a benign vascular tumor, fast-growing, which can occur in the skin, mucosa, glands and bone. In some cases it is localized in the head and neck. A more typical nasal localization appears to be the anterior portion of the nasal septum, known as locus Valsalvae or area of Little. This tumor causes a progressive nasal obstruction and recurrent epistaxis. Like all bleeding vascular tumors, hemangiomas may lead to a state of dangerous anemia in patients with heart disease. We present the case of a patient with a capillary hemangioma of the nasal septum and chronic ischemic heart disease, in treatment with antiplatelet agents, non-suspendable, and subjected to surgical removal of the tumor.
\end{abstract}

Keywords: Lobular capillary hemangioma; Endoscopic surgery; Ischemic heart disease; Embolization

\section{Introduction}

Traditionally hemangiomas are considered benign tumors of vascular nature. Some authors, however, do not have them classified as neoplasm but hamartomas or congenital vascular anomalies [1]. They grow ubiquitously in our bodies from vascular tissues in cutaneous, mucosal, bone or glandular [2]. Generally they manifest as single lesions, but in a minority of cases may be encountered, especially in childhood, multifocal diseases that accompany high risk of visceral localization, in particular intrahepatic [2]. Hemangiomas frequently affect cervical-facial districts localizing mainly to the gingiva, oral mucosa, tongue and lips [3]. Sinonasal hemangiomas are rare [1] and account for about $10 \%$ of all hemangiomas of the head

Manuscript accepted for publication December 04, 2014

${ }^{a}$ ENT, Casa di Cura Columbus, Milano, Italy

${ }^{b}$ ENT, Casa di Cura Lecco, Lecco, Italy

${ }^{\mathrm{c} C}$ Corresponding Author: Marco Capelli, Casa di Cura Columbus, Milano, Italy.

Email: info@otorinocremona.it

doi: http://dx.doi.org/10.14740/jmc2022w and neck. They mainly originate from the anterior portion of the nasal septum, very vascularized area, known as locus Valsalvae or area of Little [1] and in the nasal vestibule [2]. Also the lateral wall of the nasal cavity can be affected [4]. There are reports in the literature of intraosseous hemangioma of the inferior turbinate [5] of the maxillary sinus, the floor of the nasal cavity and the roof of the vestibule [6]. From the fifth decade sinonasal hemangiomas affect both sexes equally, while in childhood and adolescence there is a higher incidence in the male population. In the third and fourth decade, coinciding with the child-bearing age, hemangiomas are more frequently found in women [6]. It is thought that hormonal factors related to pregnancy may facilitate their development. To emphasize this association Nair et al define the septal hemangioma as a pregnancy tumor [7]. Sino-nasal hemangiomas occur primarily with unilateral nasal obstruction, recurrent epistaxis, mucopurulent rhinorrhea, epiphora, facial pain and headache or hyposmia [6]. There have also been reported cases associated with progressive painless swelling and nasal deformity [2]. The risk of bleeding and anemia is one of the main problems associated with these diseases [2]. Hemangiomas sino-nasal may present variable volumes but generally below $10 \mathrm{~mm}$ [3]; however, they can reach considerable size (even up to $80 \mathrm{~mm}$ ). These forms are called giant hemangiomas. We report the clinical case of a patient with septal emenagioma, suffering from chronic ischemic heart disease, treated with antiplatelet agents, non-suspendable, recurrent epistaxis and risk of anemia and subjected to surgical removal of the tumor.

\section{Case Report}

A 66-year-old Caucasian male with no significant family history of disease suffers from hypertension, dyslipidemia and overweight. In 1998, he is subjected to angioplasty with stenting for unstable angina. In 2010 he was hit by acute pancreatitis. In the last year he refers to the absence of angina, palpitations, dyspnea or syncope. He got treatment with metoprolol (100 mg daily), ramipril (10 mg daily), felodipine (5 $\mathrm{mg}$ daily), simvastatin (40 mg daily) and ASA (150 mg daily). He comes to our attention complaining about 1 month right nasal airway obstruction and sneezing. He has undergone nasal endoscopy showing massive ulcerated polypoid lesion (Fig. 1), brownish totally blocking the vestibular right region. This lesion seems 


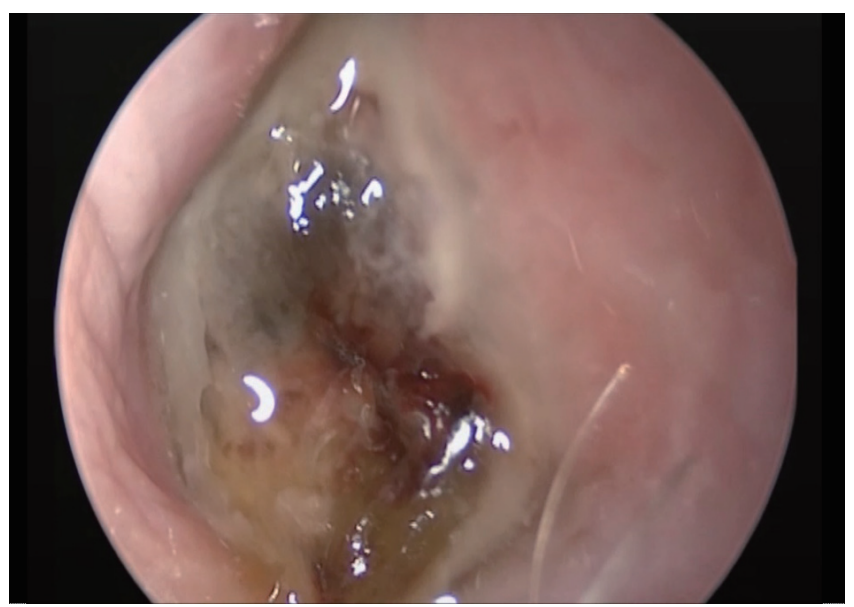

Figure 1. Endoscopic aspect of hemangioma of the nasal septum.

to originate from the locus Valsalvae. The exploration of the ipsilateral nasal cavity shows abundant mucopurulent secretions in the lower meatus. The right middle meatus and the RSE are free, and the nasopharynx is free. The endoscopic findings are normal on the left. We decide to subject the patient to oral treatment with levofloxacin $500 \mathrm{mg}$ daily and prednisone 25 $\mathrm{mg}$ for 5 days and $12.5 \mathrm{mg}$ for other 5 days. After treatment we subject the patient to CT scan of the facial bones without contrast agent and contrast-enhanced MRI of the facial bones. It confirms the presence of a polypoid lesion originating from the anterior nasal septum obstructing the vestibular region as a whole. This lesion is also richly vascularized. This lesion was approximately $2.5 \mathrm{~cm}$ in diameter. The most likely diagnosis is capillary hemangioma of the nasal septum. Because of the reported symptoms, bleeding risk associated with this disease and the possibility of anemization with worsening of the cardiac conditions, we decide to refer the patient to cardiac and echocardiographic evaluation and possible surgical excision of the mass. The patient shows cardiovascular good control and stable chronic ischemic heart disease. However, it is recommended to maintain antiplatelet therapy in the pre-, peri- and post-operative period, and this treatment is considered not to be replaced with another class of drugs. Then we reflect on the opportunity to preoperatively embolize the tumor in order to reduce the risk of surgery bleeding. We decided then, according to many authors, to waive embolization and proceed directly surgical. We subject the patient to sub-perichondral endoscopic resection of the tumor that presents the planting base on the septum. At the end of the procedure there were not recorded episodes of major bleeding, and the residual septal mucosa is of normal appearance. We place medical silicone lamina to the right fixed with a point of suture and at last we proceed to put nasal packing. Histological examination showed lesion of 2.5 $\mathrm{cm}$ compatible with capillary hemangioma ulcerated. After 10 days there is removed point of suture and silicone lamina. One month after surgery, the septal mucosa is completely healed with no evidence of synechiae. There is no evidence of residual disease or same problems in the postoperative period. At 1 year after surgery there is not observed recurrence of the disease.

\section{Discussion}

Hemangiomas of the sino-nasal district are benign vascular tumors [4]. Their origin is not well known. A review of the literature shows that possible causes are repeated trauma or microtrauma associated with prolonged nasal packing or nasal intubation [8]. Puxeddu et al $[9,10]$ provide one of the larger series of hemangiomas of the nasal cavity. In this study, we can find other possible etiological factors: pregnancy and hormonal influence. Other authors share this point of view by defining hemangioma as a pregnancy tumor [7]. However, the oncogenic viruses, an abnormal production of angiogenic growth factors or cytogenetic abnormalities could play a major role in the development of these tumors [7].

In 1982, Mulliken and Glowacki defined hemangiomas as fast-growing vascular tumors and have them classified into three subtypes: capillary hemangioma (which originates predominantly from the hypervascular septal region called locus Valsalvae), cavernous hemangioma (usually located at the side wall of the nasal cavity) [4] and mixed forms. Genc et al indicate that normally hemangiomas of nasal septum have dimensions below $10 \mathrm{~mm}$. Lesions of considerable volume occupying the entire nasal cavity are called "capillary hemangiomas giants". In the case described hemangioma, which traditionally originates from the region of the locus Valsalvae and has a diameter of about $25 \mathrm{~mm}$, occupies fully the anterior nasal region and therefore, in this case, it is correct, in our opinion, for the term of giant capillary hemangioma.

The capillary hemangiomas are macroscopically characterized by a central lobular region that histologically is formed by a network of capillary organized into lobules and a surface region which can present various aspects such as nonspecific edema, vascular dilation, inflammation and ulceration until granulation (as in our case ) $[9,11]$. The cytological structure is characterized by endothelial cells, pericytes, myeloid cells, fibroblasts and plasma cells $[2,12]$. The central lobular region, being richly vascularized, it is easily distinguishable in radiologic studies with contrast. Lee et al show some of the key aspects in the evaluation of CT hemangiomas. On CT, hemangiomas appear as masses characterized by intensity of the soft tissues, and they can capture the contrast in brightly lobular region. The surface portion, on the other hand, does not seem to take contrast. The TC is higher than any other type of radiological examination in defining the bony architecture of the sino-nasal cavity and possible cases of bone erosion that can be found at the base of the plant of the lesion. Few studies are available regarding the use of MRI in hemangiomas [9]. We consider essential an adequate radiological evaluation in the presence of a suspected lesion of vascular nature. In fact, since a biopsy is difficult for potential bleeding risks, we get a lot of data from a radiological study. Hemangiomas are to be distinguished from granulation tissue or pyogenic granulomas (formerly often wrongly confused with capillary hemangioma), vascular malformations, angiofibromas, Kaposi's sarcoma and angiosarcoma.

In the case described we highlight some critical issues to think about. Firstly, the patients suffer from a chronic heart disease, unstable angina, and he is in treatment with antiplate- 
let agents, non-suspendable. These factors may complicate the intraoperative management and may increase the intraoperative risk of bleeding. Secondly, the patient's nasal pathology can lead to repeated episodes of epistaxis until anemization. This condition would be dangerous for the maintenance of a good cardiovascular compensation in the patient concerned. Finally, the significant volume of the tumor is, once again, the potential risk of intraoperative bleeding. For these reasons, in fact, although some authors $[1,8]$ describe surgical procedures under local anesthesia, we decided to perform the surgery under general anesthesia, with the aim of maintaining controlled hypotension and reducing the risk of bleeding. This condition allowed us to maintain a clean operating field and easier endoscope management. Despite the high risk of bleeding of the case, we performed the operation without the need for preoperative embolization, according to the latest data reported in the literature [3]. Thanks to a careful sub-perichondral dissection of the mass we have not reported any significant bleeding. The use of endoscopy allowed us to identify plans of dissection very well. The nasal packing was removed on the second day. The patient did not present complications in the postoperative phase. One year after removal, he has not shown signs of recurrence.

\section{Conclusion}

Hemangioma is a common condition that affects different districts in the body. The nasal localization is believed to be rare. At this level different subtypes of hemangioma including the capillary hemangioma were identified. Like all forms of vacular tumor, hemangioma also presents some problems in the therapeutic management, especially if considerable in volume. The case described shows the very delicate planning treatment and its execution, not only because of the nasal disease but also the patient's general condition.

\section{Conflict of Interest}

The authors declare they have no financial or any conflicts of interest.

\section{References}

1. Kim ST, Kim SH, Gu GY, Cha HE. Three cases of hemangioma in nasal septum. J Rhinol. 2000;7:80-83.

2. Nedev P. Lobular capillary haemangioma of the nasal cavity in children. Trakia J Sci. 2008;6(1):63-67.

3. Genc S, Kurkcuoglu SS, Karabulut H, Acar B, Tuncel U, Degerli S. Giant lobular capillary hemangioma of the nasal septum. Turk J med Sci. 2009;39(2):325-328.

4. Archontaki M, Stamou AK, Hajiioannou JK, Kalomenopoulou M, Korkolis DP, Kyrmizakis DE. Cavernous haemangioma of the left nasal cavity. Acta Otorhinolaryngol Ital. 2008;28(6):309-311.

5. Akiner MN, Akturk MT, Demirtas M, Atmis EO. Intraosseous cavernous hemangioma of inferior turbinate: a rare case report. Case Rep Otolaryngol. 2011;2011:431365.

6. Budiman BJ, Octiza R. Capillary haemangioma of nasal septum.

7. Nair CS, Bahal MA, Bhadauria RS. Lobular capillary hemangioma of nasal cavity. MJAFI. 2008;64:270-271.

8. Fasunla AJ, Adebola OS, Okolo CA, Adeosun AA. Nasal septal lobular capillary haemangioma in West Africa subregion: a case report. Cases J. 2009;2:8952.

9. Lee DG, Lee SK, Chang HW, Kim JY, Lee HJ, Lee SM, Kwon JH, et al. CT features of lobular capillary hemangioma of the nasal cavity. AJNR Am J Neuroradiol. 2010;31(4):749-754.

10. Puxeddu R, Berlucchi M, Ledda GP, Parodo G, Farina D, Nicolai P. Lobular capillary hemangioma of the nasal cavity: A retrospective study on 40 patients. Am J Rhinol. 2006;20(4):480-484.

11. Toida M, Hasegawa T, Watanabe F, Kato K, Makita H, Fujitsuka H, Kato Y, et al. Lobular capillary hemangioma of the oral mucosa: clinicopathological study of 43 cases with a special reference to immunohistochemical characterization of the vascular elements. Pathol Int. 2003;53(1):1-7.

12. Mills SE, Cooper PH, Fechner RE. Lobular capillary hemangioma: the underlying lesion of pyogenic granuloma. A study of 73 cases from the oral and nasal mucous membranes. Am J Surg Pathol. 1980;4(5):470-479. 